\title{
Molecular evaluation of Toxocara infection in stray cats using loop-mediated isothermal amplification (LAMP) technique as a rapid, sensitive, and simple screening assay
}

Hamid Azimian

Lorestan University

Hamidreza Shokrani

Lorestan University

Shirzad Fallahi ( $\sim$ Shfupdate@gmail.com )

Lorestan University of Medical Sciences https://orcid.org/0000-0003-1826-7910

\section{Research Article}

Keywords: Toxocara species, Stray cats, LAMP technique, Khorramabad

Posted Date: May 22nd, 2020

DOl: https://doi.org/10.21203/rs.3.rs-29562/v1

License: (9) (i) This work is licensed under a Creative Commons Attribution 4.0 International License.

Read Full License 


\section{Abstract}

Background: Toxocara species are parasitic nematodes of dogs and cats with a worldwide distribution. The adult worm lives in the intestine of dogs and cats, and infection occurs through eating paratenic host or embryonated eggs. These nematodes are considered as one of the common pathogens of humans and livestock, and in humans cause Visceral larva migrans (VLM) syndrome, which is accompanied by different clinical symptoms, sometimes lead to respiratory problems and even death. The present study aimed to investigate the molecular frequency of Toxocara species in stray cats using the loop-mediated isothermal amplification (LAMP) technique.

Methods: During 3 months (July to September 2018) a total of 95 stool samples were randomly collected from stray cats in Khorramabad city, Lorestan province, West of Iran. To separate the parasite's eggs, the flotation method with chlorine solution was applied to saturation. Microscopic examination was performed after the separation and extraction of supernatants. To rupture the four layers of Toxocara egg, and extraction of DNA, sonication and freeze and thaw processes were used. After DNA extraction from the stool specimens, the LAMP reaction was performed using the internal transcribed spacer 2 (ITS2) gene primers of Toxocara species and the appropriate LAMP master mix.

Results: The overall prevalence of Toxocara spp. in stray cats of Khoramabad city was $20 \%(19 / 95)$ by parasitological and molecular assessments. The microscopic examination of stool samples revealed that 19 samples were positive for Toxocara spp. The same 19 positive samples were also positive in the LAMP experiments. Interestingly, based on the results of the LAMP assay, out of 95 studied samples, 18 (18.41\%) specimens were belonged to Toxocara canis, while only $1(0.5 \%)$ sample was diagnosed as Toxocara cati.

Conclusion: Identification of Toxocara spp. in dogs and cats as the important sources of human infections especially for children is particularly important. The relatively high prevalence of Toxocara species, especially and interestingly the canis species in the studied cats shows the role of these animals in spreading the disease and the risk of transmission of infection to humans. Precautionary measures such as preventing cats from traveling in public places, especially children's playgrounds, as well as avoiding direct contact with stray cats are recommended.

\section{Background}

Toxocara spp. (Toxocara canis, Toxocara cati and Toxascaris leonine) are parasitic roundworms (nematodes) of predatory mammals from families Canidae and Felidae with worldwide distribution [1, 2]. T. canis is one of the most widespread public health and economically important zoonotic parasitic infections humans share with dogs, cats, and wild canids, particularly foxes and jackals [3]. Definitive hosts of $T$. cati are wild and domestic felids [2]. The adult worm lives in the intestine of dogs and cats, and infection in humans is caused by eating embryonated eggs or meat from the paratenic host, including cattle, sheep, and chicken [4]. The life cycle of T. cati is like T. canis, with the difference that 
prenatal contamination does not occur in $T$. cati and there is no congenital infection. When the eggs are excreted, they are not immediately containing larvae, but it takes 3-4 weeks to form the larva and become infectious [5]. The highest prevalence of Toxocara is seen in kittens and puppies at the age of 24 weeks. Puppies may be infected by breastfeeding or through the umbilical cord during fetal development, and kittens are also infected through breastfeeding [6, 7]. The zoonosis disease resulted from these nematodes is called toxocariasis. Infection in humans, especially in children, occurs through eating parasite eggs when playing with soils contaminated with dog and cat feces [8]. These nematodes have a wide range of paratenic hosts, including rodents, birds, humans, and mammals. In the body of these hosts, Toxocara larvae migrate to different organs and cause serious complications [9]. There are reports that toxocariasis has been created in humans after eating raw or under-cooked liver and meat of chickens, ducks, pigs and lambs $[9,10]$. Although Toxocara species do not grow and mature in the human body, however, contamination with the second stage larvae (L2) can lead to serious complications such as high eosinophilic visceral larvae migrnas (VLM), hepatomegaly, pulmonary involvement with signs of chronic pulmonary inflammation associated with cough and fever which can sometimes lead to breathing problems and even death. Additionally, toxocariasis may also cause ocular larvae migrnas (OLM) with eye problems as well as epilepsy and myocarditis [11-13]. Toxocara is a globally soiltransmitted nematode and human infection has been reported in almost all regions of the world, especially those with tropical weather and abundance of dogs and cats. This parasite is spread not only in developing countries but also in all developed countries so that in some of these countries it is the most common human helminthic infection. Human infections are more commonly reported in children, especially children of low age $[11,14]$. Keeping dogs (especially puppies) and cats as a pet and touching these animals, soil eating (pica) by children, free entry of dogs and cats into farmland and public parks, and non-compliance with sanitation in parasitizing raw vegetables are among the most important risk factors associated with toxocariasis [6].

Toxocara infection can be detected in dogs and cats using history and based on clinical signs. Finding the brown eggs with thick and hollow walls in the fecal specimen determine the diagnosis. The wet mount (direct smear) preparation from the stool specimen solely is not an appropriate method for finding eggs while accompanied by the concentration methods such as flotation assays make a more accurate diagnosis [15]. The sensitivity and specificity of the floatation method for detecting Toxocara eggs were $51 \%$ and $100 \%$, respectively. Positive and negative results are also $100 \%$ and $81 \%$ reliable respectively [16].

The use of new diagnostic methods, such as molecular assays, to determine the rate of infection in dogs and cats as reservoirs of Toxocara, is applicable and can be compared to the results of other tests, including routine stool examination. The loop-mediated isothermal amplification (LAMP) technique is a simple high-performance method that was introduced by Notomi et al. in 2000, in which the DNA replicated specifically, efficiently and rapidly under isothermal conditions [17-20]. In this method, four specifically designed primers (two internal primers and two external primers) which totally identify six regions among the target DNA are used. The target region is replicated during the comet process, forming a series of the loop in a temperature of $65-60^{\circ} \mathrm{C}$ by using a heat-resistant DNA polymerase enzyme (Bst 
DNA polymerase).. The LAMP technique is a simple method that does not require expensive equipment such as Thermal cycler and Gel documentation systems and the reaction can be done in a hot water bath or thermal block [19-21]. The results can be simply evaluated by visual examination of color change of SybrGreen I, an intercalating dye that specifically binds to the double-stranded DNA in reaction tubes from orange to green fluorescence under daylight and UV light [22-24].

The high population of stray cats, as one of the definitive hosts for Toxocara, in Khorramabad city, few studies conducted in this area, and the high resistance of Toxocara eggs, as a major source of environmental contamination, shows the necessity of research on the rate of infection in stray cats in Khorramabad, Western Iran.

\section{Methods}

\section{Samples collection}

During a 3-months period (July to September 2018), a total of 95 stool samples were randomly collected from stray cats in Khorramabad city, Lorestan province, West of Iran. Samples were immediately transferred to the Parasitology Laboratory Faculty of Medicine, Lorestan University of Medical Sciences and divided into two sections. One part of each specimen was kept at $-20^{\circ} \mathrm{C}$ for DNA extraction and subsequent molecular evaluation, and the other part was prepared for parasitological examinations.

\section{Parasitological examinations}

At first, in order to homogenize, the stool specimens were poured into a tube containing normal saline $(0.85 \% \mathrm{NaCl}$ solution) and vortex for 5 minutes. Then the homogenize samples were examined by microscopic observation (Zeiss, Germany, $100 \times$ and 400x magnification) of direct smears using normal saline, and floatation with a chlorine solution. To separate the excess materials, the suspensions were passed through two layers of wet tampons. Thereafter, the samples were centrifuged at $3000 \times \mathrm{g}$ for 3 minutes, then the supernatant was discarded and the chlorine was spilled over the saturation into the tube and centrifuged at $2000 \times \mathrm{g}$ for 5 minutes. Then $300 \mu \mathrm{L}$ of the supernatant solution was removed and poured into a $1.5 \mathrm{ml}$ microtube. The Toxocara eggs were identified by microscopic observation of supernatant under $100 \times$ and $400 \times$ magnification.

\section{Sonication and freeze and thaw process}

In order to rupture the four layers of Toxocara eggs and extraction of DNA, the sonication and freeze and thaw methods were used. First, the cycle of sonication machine was set to 0.5 and the voltage was set to 70. The microtube containing the specimens was placed inside the ice and subsequently, each sample was sonicated for 20 seconds ( 5 times every 4 seconds). For the freezing and thawing process, the samples were placed 5 times every 2 minutes inside the liquid nitrogen and water bath at $95^{\circ} \mathrm{C}$. 


\section{Molecular examination}

DNA extraction from the samples was performed using the DNA extraction kits (Yekta Tajhiz Azma Co. Iran) based on the protocol of the manufacturer. The LAMP technique was performed on the basis of the highly conserved internal transcribed spacer 2 (ITS2) gene of Toxocara spp. The nucleotide sequences of primers used in the LAMP reaction are shown in Table 1 [25]. The reaction was carried out in a final volume of $25 \mu \mathrm{l}$ as previously was described [18]. Due to the fact that the loop primers were not designed for the ITS2 gene of both T. canis and T. cati, in the LAMP reaction the double-distilled water was replaced. To the visual assessment of the LAMP amplicons, three microliters of SYBR Green I (Invitrogen, Thermo Fisher Scientific Invitrogen lot, Carlsbad, California, United States) diluted in DMSO (Dimethyl sulfoxide), was added to each tube and observed under daylight and UV light. Furthermore, the gel electrophoresis was performed on the LAMP products in a $1.5 \%$ agarose gel stained with DNA safe stain $(1 \mu \mathrm{g} / \mathrm{mL})$ and visualized under UV light. The assay reproducibility was evaluated by the duplicated reactions of the samples simultaneously. As positive and negative controls, the genomic DNA from Toxocara spp. standard strain and doubled distilled water were also included in each LAMP reaction.

Table 1

The nucleotide sequence of primers targeting the ITS2 gene of Toxocara spp. used in the LAMP reaction (Macuhova 2010).

\begin{tabular}{|lll|}
\hline Target spp. & Primer set & Primer Sequence $\left(\mathbf{5}^{\prime} \mathbf{-} \mathbf{3}^{\prime} \mathbf{)}\right.$ \\
& & \\
& Tcati-F3 & ccacgtaccttgccaagac \\
& Tcati-B3 & gcgcattccttcttcaagca \\
T. cati & Tcati-FIP & ggaacacatacgccaatggccatgcacaagaatcgctgtcg \\
& Tcati-BIP & acgatatggcctccagcaagccgatgacgttacctccaacc \\
& & \\
& Tcan-F3 & tgtgattaacgcgcaaggt \\
& Tcan-B3 & ctggaggccgtatcgtga \\
& Tcan-FIP & ccttggcaaggtacgctgtacatgtggtgcattcggtgag \\
& Tcan-BIP & tcgcacaagaatggctgtcgtagcaacgcaacatacactca \\
& & \\
\end{tabular}

\section{Results}

\section{Parasitological examinations}


Based on parasitological examinations of the stool specimens by direct smears (wet mount) and floatation with a chlorine solution, the frequency of Toxocara spp. was 20\% (19/95) among stray cats of Khorramabad, western Iran. The number of positive samples detected by the flotation method was approximate twice the number of positive samples detected in the direct smears method (12 and 7 samples respectively). (Data not shown)

\section{Sonication and freeze and thaw process}

In order to rupture the different layers of Toxocara eggs and facilitate the extraction of DNA, the process of sonication and freezing and thawing of the samples was performed. To ensure the destruction of egg layers, this process was performed on a microscopic positive specimen with a large number of Toxocara eggs (Fig.1). After microscopic re-examination, the degraded layers of broken eggs were observed.

\section{Molecular examination}

In this study, CybrGreen I intercalating fluorescent dye was used to evaluate the LAMP results. The color change from orange to brilliant green indicates a positive result while the negative result will remain as orange as it is (Fig. 2 and 4). To confirm the ultimate diagnosis, positive samples were electrophoresed on $1.5 \%$ agarose gel. Positive samples appear in smear and ladder-like view with multiple bands and different sizes on the agarose gel (Fig. 3 and 5). Based on the results of the LAMP reaction, out of 95 studied samples, 18 samples (18.49\%) were infected with T. canis (Fig. 2 and 3 ) while only one sample (0.5\%) was infected with T. cati (Fig. 4 and 5).

\section{Discussion}

Due to the harmful effects of toxocariasisinfection in humans such as high eosinophilic VLM syndrome, hepatomegaly, pulmonary involvement, OLM syndrome, myocarditis, epilepsy and even death caused by the second stage larvae (L2) of the parasite, detection of Toxocara spp. is important in dogs and cats as important sources of human infection, especially for children $[13,26]$. Based on the results of the present study, 19 samples $(20 \%)$ of 95 fecal specimens of the studied cats were infected with Toxocara spp. Which interestingly 18 cases $(18.94 \%)$, belonged to $T$. canis while only one case $(1.5 \%)$ was diagnosed as $T$. cati. These results show the relatively high prevalence of Toxocara species especially $T$. canis in stray cats in Khorramabad western Iran, indicates the potential of these animals as an important reservoir for the transmission of infection to humans, especially children. The results of this study are consistent with the results of most studies conducted in different regions of Iran on cats in terms of a relatively high prevalence of Toxocara spp. [27-31]. However, these studies are different from the current study in view of the abundance of Toxocara species, and contrary to the results of most of these studies, the dominant species in this study was $T$. cati. Such a result could be due to the high prevalence of stray dogs in this area, high contamination of the environment and possibly the transmission of infection to stray cats and, consequently, the higher prevalence of canis species among the studied cats. There have been two 
reports from Malaysia of a 'variant' ascaridoid of cats assigned to the species T. canis [3, 32]. Lee and colleagues (1993) reported that of the 55 cats collected from around Kuala Lumpur, Malaysia, 15 cats were infected with Toxocara spp. of these, 12 cats were infected with T. cati and the others were infected with $T$. canis [3]. Likewise, Parsons and colleagues reported a disseminated granulomatous disease in a cat caused by larvae of $T$. canis [33]. In another study, they also examined the pathological and haematological responses in cats experimentally infected with T. canis larvae [34]. In a study conducted by zibaei et al. (2010) on 285 soil samples from public parks in Khorramabad, $63.3 \%$ of samples were contaminated with Toxocara eggs using parasitology assessments [35]. The result of this study showed the high environmental contamination caused by the high infection rate in dogs and cats in Khorramabad city. In the present study, there was no difference between the results of microscopy and the LAMP technique and a perfect concordance was observed between LAMP assay and microscopic examination on the cat's feces for Toxocara detection. Similar to the results of the present study, no difference was found between the results of the microscopic method and 2qPCR technique, a specific and rapid duplex quantitative real-time PCR on the ITS -2 gene of Toxocara that was used by Durant et al. 2012 to detect $T$. cati and T. canis in soil and feces samples [8]. In a study by Ozlati et al. 2016 in Tabriz city, Northwest Iran, 180 samples of suspected contaminated soil were studied using microscopic, PCR and LAMP methods. Based on the results, 57, 14 and 77 samples were detected positive for Toxocara by microscopy, PCR and LAMP methods respectively, of which 49 samples $(27.2 \%)$ were contaminated with $T$. cati and 28 samples (15.5\%) were contaminated with $T$. canis. The results of Ozlati and colleagues showed a higher sensitivity of the LAMP technique than the PCR and microscopy methods [36].

The difference in the prevalence of Toxocara in different regions depends on several parameters including the population of dogs, cats, and native rodent of the study area, the public health conditions, the season in which the study was carried out, and the type of diagnostic method applied for parasite detection [31]. The development of parasite eggs in environment and becoming infectious, depends on temperature and humidity of the environment and an area with higher temperature and humidity, this process accelerates faster and the dogs and cats as the definitive hosts, become more infected and subsequently the prevalence of infection is greater. In the area with a high population of dogs and cats as well as the poor public health conditions, the prevalence of Toxocara spp. is also higher. Mice as the main paratenic host of the parasite have an important role in its prevalence.

Studies in different parts of the world also showed a high prevalence of Toxocara species in the main hosts and different environments. Macuhova et al. 2010 in Japan, used the LAMP and PCR technique targeting the ITS -2 gene of Toxocara to diagnose the T. cati and T. canis in 5 sand samples, their results showed that 4 out of 5 samples were contaminated with $T$. cati, and there was no contamination with $T$. canis [25]. After calving and testing feces on 58 cats by Calvete et al. (1998), in Spain, 55.2\% of the cats were diagnosed infected with T. cati [37]. In a study by Borthakur and Murkharjee, (2011) on stray cats in Mizoram, India, using parasitology assays, $85.2 \%$ of the cats were infected with various parasites, and $T$. cati was the second most commonly detected parasites after Taenia formis [38]. In 2015, Hussam and Aredhi investigated fecal samples of feline cats in Iraq, of which $47.77 \%$ were infected with intestinal parasites and the prevalence of T. cati was $23.25 \%$ [39]. 


\section{Conclusions}

Identification of Toxocara species in dogs and cats is important because it is an important source of human infections especially for children, and the second phase of the larvae causes VLM and OLM syndromes in humans. The relatively high prevalence of Toxocara species in the studied cats shows the potential role of these animals in spreading the disease and the risk of transmission to humans. According to the results of this study, it is necessary to take preventive measures, including preventing cats from traveling in public places, especially children's playgrounds, and avoiding direct contact with stray cats.

\section{Abbreviations}

Bst DNA polymerase: Bacillus stearothermophilus DNA Polymerase; ITS2: internal transcribed spacer 2; LAMP: loop-mediated isothermal amplification; L2: second stage larvae; OLM: ocular larvae migrnas; VLM: visceral larva migrans.

\section{Declarations}

\section{Acknowledgements}

We thank the staff of the Razi Herbal Medicines and Parasitology Research Centers, Lorestan University of Medical Sciences, Khorramabad, Iran for their assistance to collect and examine the samples. This article is derived from the Doctor of Veterinary Medicine's thesis of the First author (Thesis No. 982), Department of Pathobiology, Faculty of Veterinary Medicine, Lorestan University, Khorramabad, Iran

\section{Authors' contributions}

SHF: project development, perform the experiments, manuscript writing and editing; HA: data collection, perform the experiments; $\mathrm{HSH}$ : conceptualization, formal analysis, manuscript writing and editing.

\section{Authors' information}

Sh-F is the corresponding authors of this paper of this article.

\section{Funding}

This research was financially supported by Lorestan University, Khorramabad, Iran (A-10-1603-2).

\section{Availability of data and materials}


The datasets used and/or analyzed during the current study are available from the corresponding author on reasonable request.

\section{Ethics approval and consent to participate}

The present study was approved by the Ethics Committee of Lorestan University (IR.LU.REC.1396.195) and ethical considerations regarding to work with animals was observed.

\section{Consent for publication}

Not applicable.

\section{Competing interests}

The authors declare that they have no conflict of interest.

\section{References}

1. Eslami AS. Veterinary helminthology, Tehran University Press. 2006; 3: 129-45.

2. Okulewicz A, Perec-Matysiak K, Buńkowska JH. Toxocara canis, Toxocara cati and Toxascaris leonina in wild and domestic carnivores. Helminthologia 2012; 49: 3-10.

3. Lee CC, Cheng NY, Bohari Y. Toxocara canis from domestic cats in Kuala Lumpur. Trop Biomed. 1993; 10: $79-80$.

4. Acosta L, León-Quinto T, Bornay-Llinares FJ, Simón MA, Esteban JG. Helminth parasites infaecal samples from theendangered Iberianlynx (Lynx pardinus). Vet Parasitol. 2011; 179: 175-9.

5. Fisher M. Toxocara cati: an underestimated zoonotic agent. Trends Parasitol. 2003; 19: 167-70.

6. Rubinsky-Elefant G, Hirata CE, Yamamoto JH, Ferreira MU. Human toxocariasis: diagnosis, worldwide seroprevalences and clinical expression of the systemic and ocular forms. Annals Trop Med Parasitol. 2010; 104: 3-23.

7. Athari A. Marcell and Vogue Medical Parasitology. Markla A, Ka, John TJ, Croat W, Ninth Edition, Tehran, AYJ Publishing 2010.

8. Durant JF, Irenge LM, Wyrwas RF, Dumont C, Doucet JP, Mignon B, et al. Duplex quantitative real-time PCR assay for the detection and discrimination of the eggs of Toxocara canis and Toxocara cati (Nematoda, Ascaridoidea) in soil and fecal samples. Parasit Vectors. 2012; 7: 288. 
9. David TJ, Petrie W. Markel and Vogue Medical Parasitology. Translation of Amharad Azhari, TehranAyed Publishing, 2007; 398-401.

10. Maruyama S, Nino T, Yamamoto Katsube Y. Parasitism of Toxocara canis larvae in chickens inoculated with the Ascarid eggs. J Vet Med Sci. 1994; 56: 139-41.

11. Glickman LT, Schantz PM. Epidemiology and Pathogenesis of Zoonotic Toxocariasis. Epidemiol Rev. 1981; 3: 230-50.

12. Alavi SM, Sefidgaran G. Frequency of anti Toxocara antibodies in school children with chronic cough and eosinophilia in Ahvaz, Iran. Pakistan J Med Sci. 2008; 24: 360-3.

13. Kumagai T, Shimogawara FR, Ohmae H, Wang TP, Lu S, Chen R, et al. Detection of early and single infections of Schistosoma japonicum in the intermediate snails, Oncomelania hupensis, by PCR and loopmediated isothermal amplification (LAMP) assay. American J Trop Med Hyg. 2010; 83: 542-8.

14. Despommier D. Toxocariasis: clinical aspects, epidemiology, medical ecology, and molecular aspects. Clin Microbiol Rev. 2003; 16: 265-72.

15. Dryden M. Diagnosis and control of gastro-intestinal parasites in dogs and cats. Vet Quart. 1996; 18: $42-3$.

16. Overgaauw PAM. Prevalance of intestinal nematodes of dogs and cats in the Netherlands. Vet Quart. 1997; 19: 7-14.

17. Notomi T, Okayama H, Masubuchi H, Yonekawa T, Watanabe $\mathrm{K}$, Amino N, et al. Loop-mediated isothermal amplification of DNA. J Nucleic Acids Res 2000; 28: 63.

18. Fallahi Sh, Seyyed Tabaei SJ, Pournia Y, Zebardast N, Kazemi B. Comparison of loop-mediated isothermal amplification (LAMP) and nested-PCR assay targeting the RE and B1 gene for detection of Toxoplasma gondii in blood samples of children with leukaemia. Diag Microbiol Infect Dis. 2014; 79 : 347-54.

19. Fallahi Sh, Mazar Z, Ghasemian M, Haghighi A. Challenging loop-mediated isothermal amplification (LAMP) technique for molecular detection of Toxoplasma gondii. Asian Pacific J Trop Med. 2015; 8: 366-72.

20. Fallahi Sh, Moosavi SF, Karimi A, Chegeni AS, Saki M, Namdari P, et al. An advanced uracil DNA glycosylase-supplemented loop-mediated isothermal amplification (UDG-LAMP) technique used in the sensitive and specific detection of Cryptosporidium parvum, Cryptosporidium hominis, and Cryptosporidium meleagridis in AIDS patients. Diag Microbiol Infect Dis. 2018; 91: 6-12.

21. Hanifehpour H, Samsam Shariat SK, Ghafari MS, Kheirandish F, Saber V, Fallahi Sh. Serological and molecular diagnosis of Toxoplasma gondii infections in thalassemia patients. Iranian J Parasitol. 2019; 
14: $20-8$.

22. Kaneko H, Kawana T, Fukushima E, Suzutani T. Tolerance of loop-mediatedisothermal amplification to a culture medium and biological substances. J Biochem Biophys Method. 2007; 70: 499-501.

23. Nagamine K, Watanabe K, Ohtsuka K, Notomi T. Loop-mediated isothermal amplification reaction using a nondenatured template. J Clin Chem. 2001; 47: 1742-3.

24. Mori Y, Hirano T, Notomi T. Sequence specific visual detection of LAMP reactions by addition of cationic polymers. J BMC Biothecnol. 2007; 6: 1-10.

25. Macuhova K, Kumagai T, Akao N, Ohta N. Loop-Mediated Isothermal Amplification Assay for Detection and Discrimination of Toxocara canis and Toxocara cati Eggs Directly from Sand Samples. J Parasitol. 2010; 96: 1224-7.

26. Kleine A, Springer A, Strube C. Seasonal variation in the prevalence of Toxocara eggs on children's playgrounds in the city of Hanover, Germany. Parasite Vectors. 201; 10: 248.

27. Changizi E, Mobedi I, Salimi-bajestani MR, Rezaie-dust A. Gastrointestinal Helminthic Parasites in Stray Cats (Felis catus) from North of Iran. Iranian J Parasitol. 2007; 2: 25-9.

28. Sharif M, Nasrolahei M, Ziapour S, Gholami S, Ziaei H, et al., Toxocara cati infections in stray cats in northern Iran. J Helminthol. 2007; 81: 63-6.

29. Arbabi M, Hooshyar H. Gastrointestinal parasites of stray cats in Kashan, Iran. Trop Biomed. 2009; 26: $16-22$.

30. Khademvatan Sh, Fakher R, Tavalla M, Abdizadeh R, Hashemitabar M. PCR-Based Molecular Characterization of Toxocara spp. Using Feces of Stray Cats: A Study from Southwest Iran. PLoS One 2013; 8: e65293.

31. Torkan S, Ghandehari-Alavijeh MR, Khamesipour F. Survey of the prevalence of Toxocara cati in stray cats in Isfahan city, Iran by PCR method. Trop Biomed. 2017; 34: 550-5.

32. Rohde H. Helminths of cats and dogs in Malaya. In UNESCO-First Regional Symposium on Scientific Knowledge of Tropical Parasites, University of Singapore. 1962; 207-16.

33. Parsons JC, Bowman DD, Grieve RB. Disseminated granulomatous disease in a cat caused by larvae of Toxocara canis. J Comp Path. 1988; 99: 343-6.

34. Parsons JC, Bowman DD, Grieve RB. Pathological and haematological responses of cats experimentally infected with Toxocara canis larvae. Int J Parasitol. 1989; 19: 479-88.

35. Zibaei M, Abdollahpour F, Birjandi M, Firoozeh F. Soil contamination with Toxocara spp. eggs in the public parks from three areas of Khorram Abad, Iran. Nepal Med Colleg J. 2010; 12: 63-5. 
36. Ozlati M, Spotin A, Shahbazi A, Mahami-Oskouei M, Hazratian T, Adibpor M, et al. Genetic variability and discrimination of low doses of Toxocara spp. from public areas soil inferred by loop-mediated isothermal amplification assay as a field-friendly molecular tool. Vet World. 2016; 9: 1471.

37. Calvete C, Lucientes J, Castillo JA, Estrada R, Gracia MJ, Perib`an ez MA, et al. Gastrointestinal helminth parasites in stray cats from the mid-Ebro Valley, Spain. Vet Parasitol. 1998; 75: 235-40.

38. Borthakur Sk, Murkharjee SN. Gastrointestinal Helminthes In Stray Cats (Felis Catus) From Aizawl, Mizoram, India. Southeast Asian J Trop Med Public Health. 2011; 42: 255-8.

39. Hussam S, Aredhi A. Prevalence of gastrointestinal parasites in domestic cats (Feliscatus) in AlDiwaniya province / Iraq. Int J Current Microbiol Applied Sci. 2015; 4: 166-71.

\section{Figures}

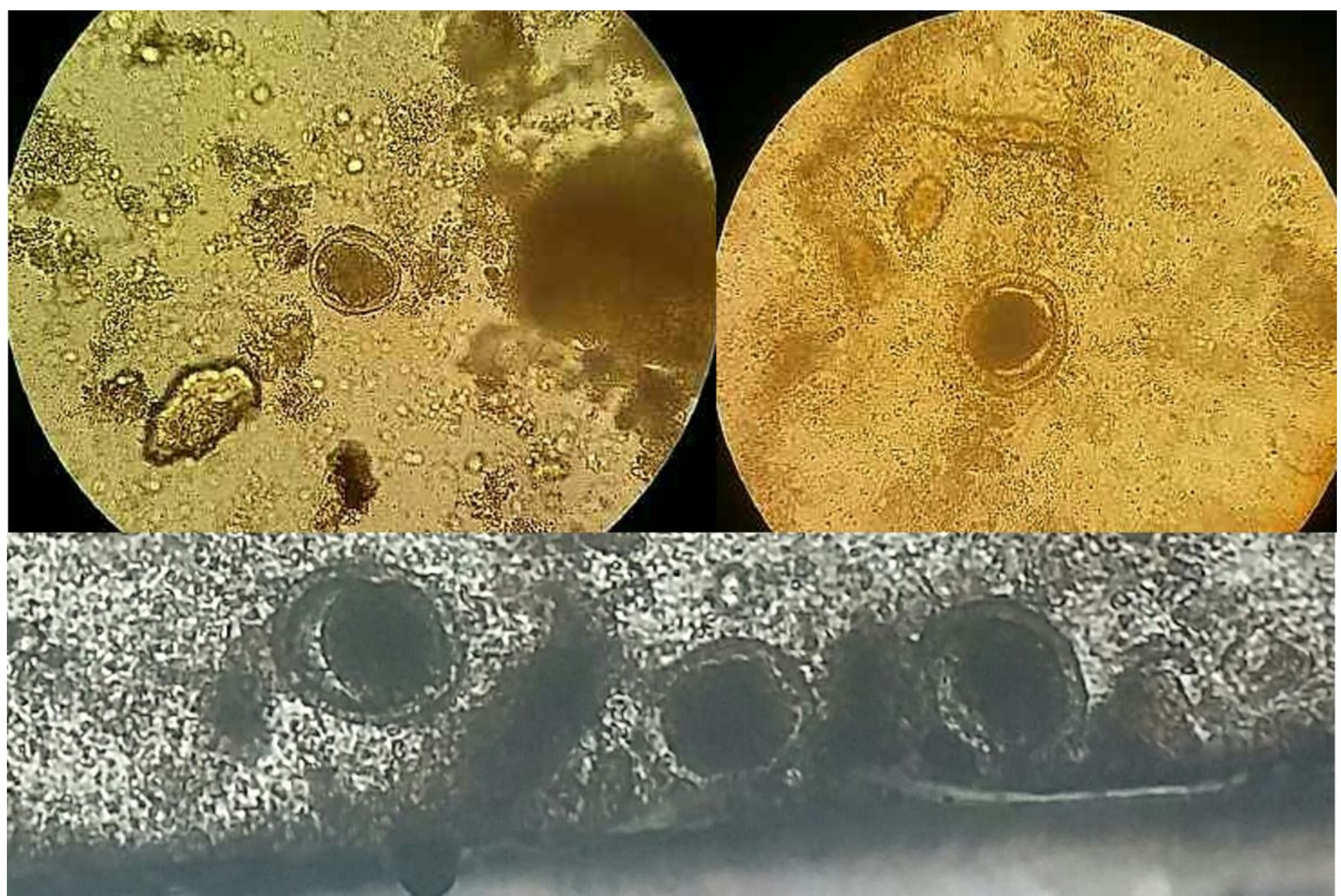

\section{Figure 1}

Microscopic evaluation of the positive stool specimens containing Toxocara eggs 


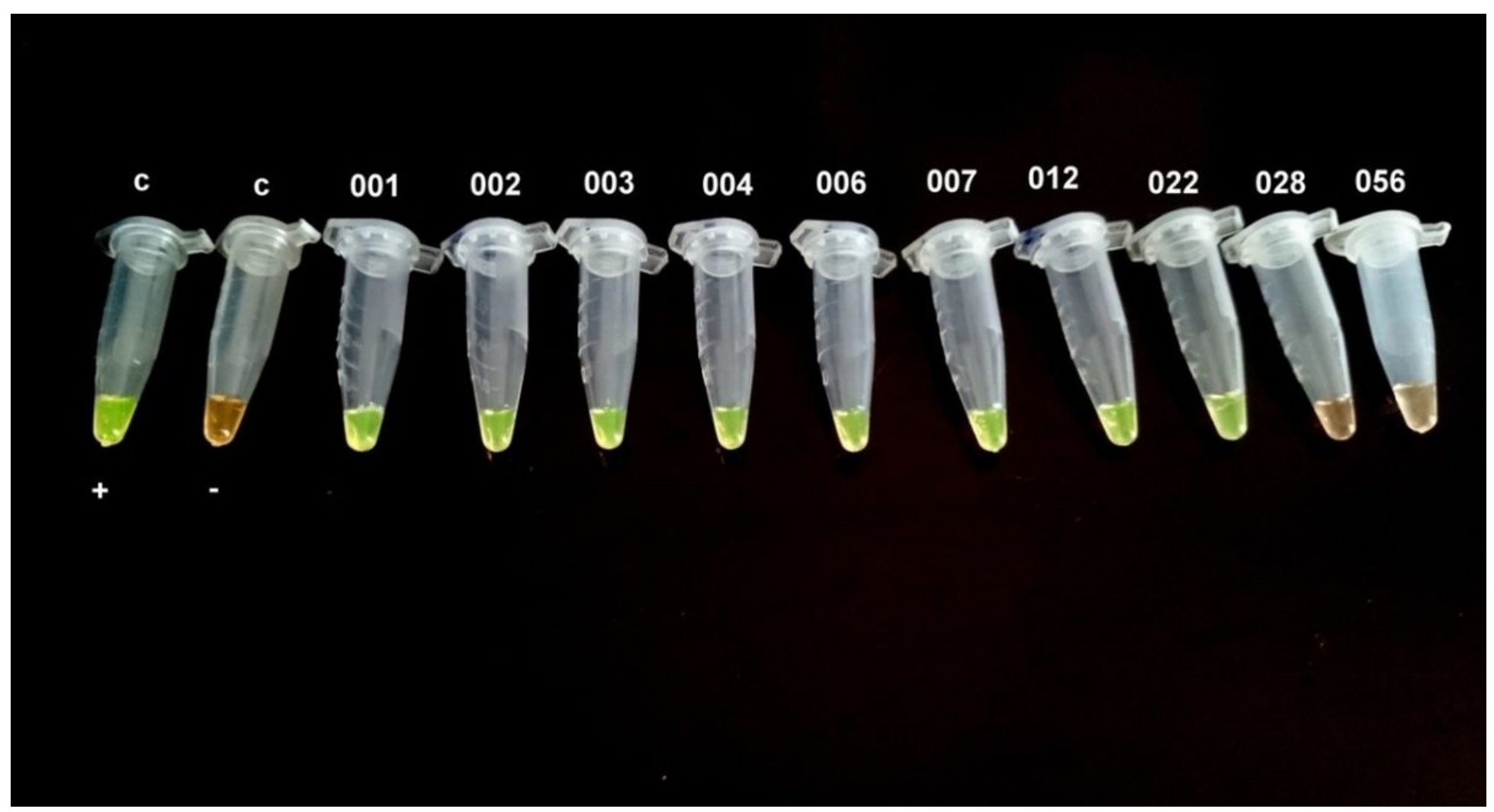

Figure 2

Analysis results of LAMP on stool samples from stray cats using SYBR Green I DNA stain under UV light. C+; Positive control, C-; Negative control, microtubes 001, 002, 003, 004, 006, 007, 012, and 022; represent the positive LAMP results for T. canis in the DNA samples from stray cat's feces 


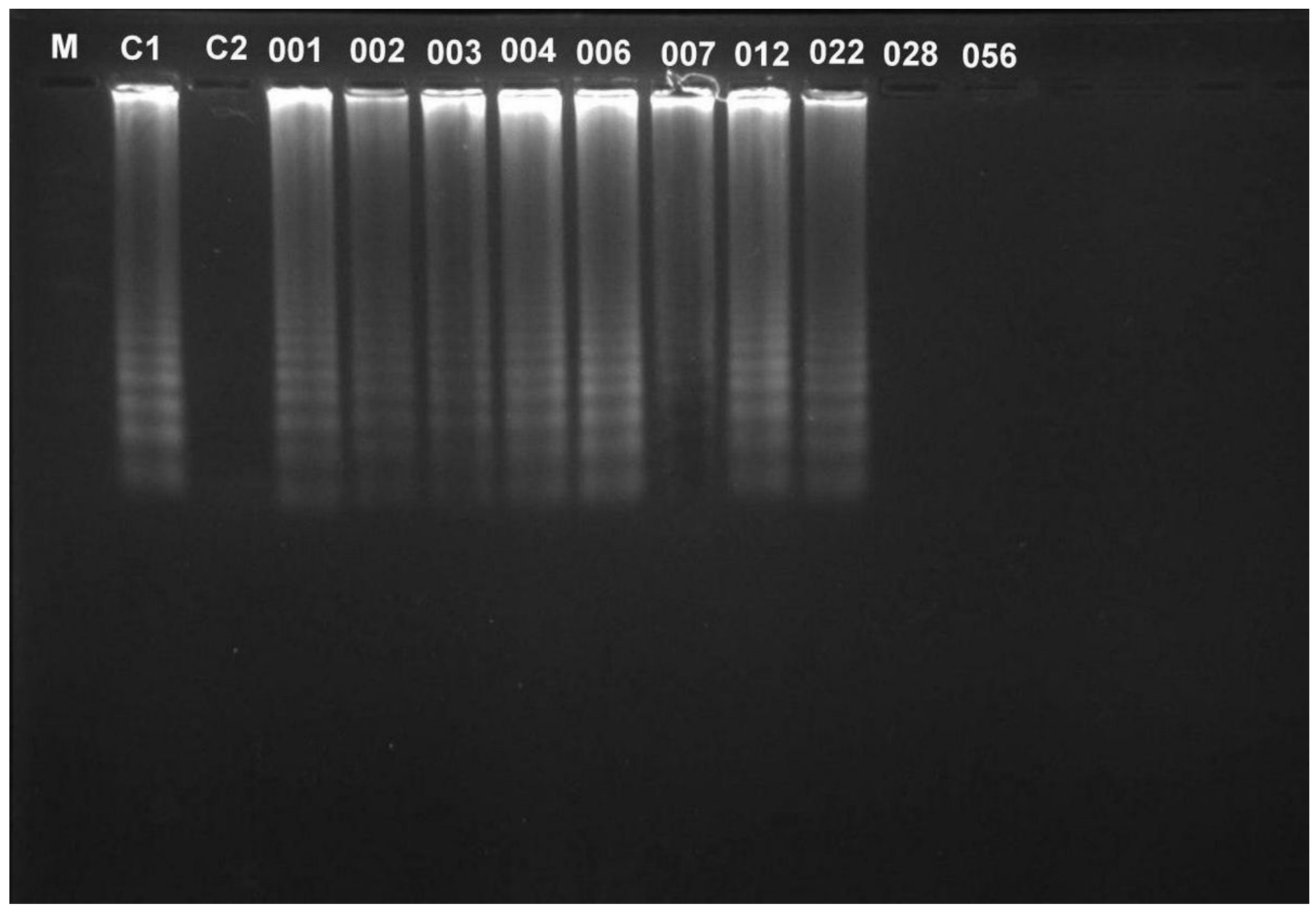

Figure 3

Evaluation of LAMP products by electrophoresis on 1.5\% agarose gel stained with SYBR Safe DNA gel stain. M; 100 bp molecular weight marker, C1; Positive control, C2; Negative control, lanes 001, 002, 003, $004,006,007,012$, and 022; represent the positive LAMP results for T. canis in the DNA samples from stray cat's feces 


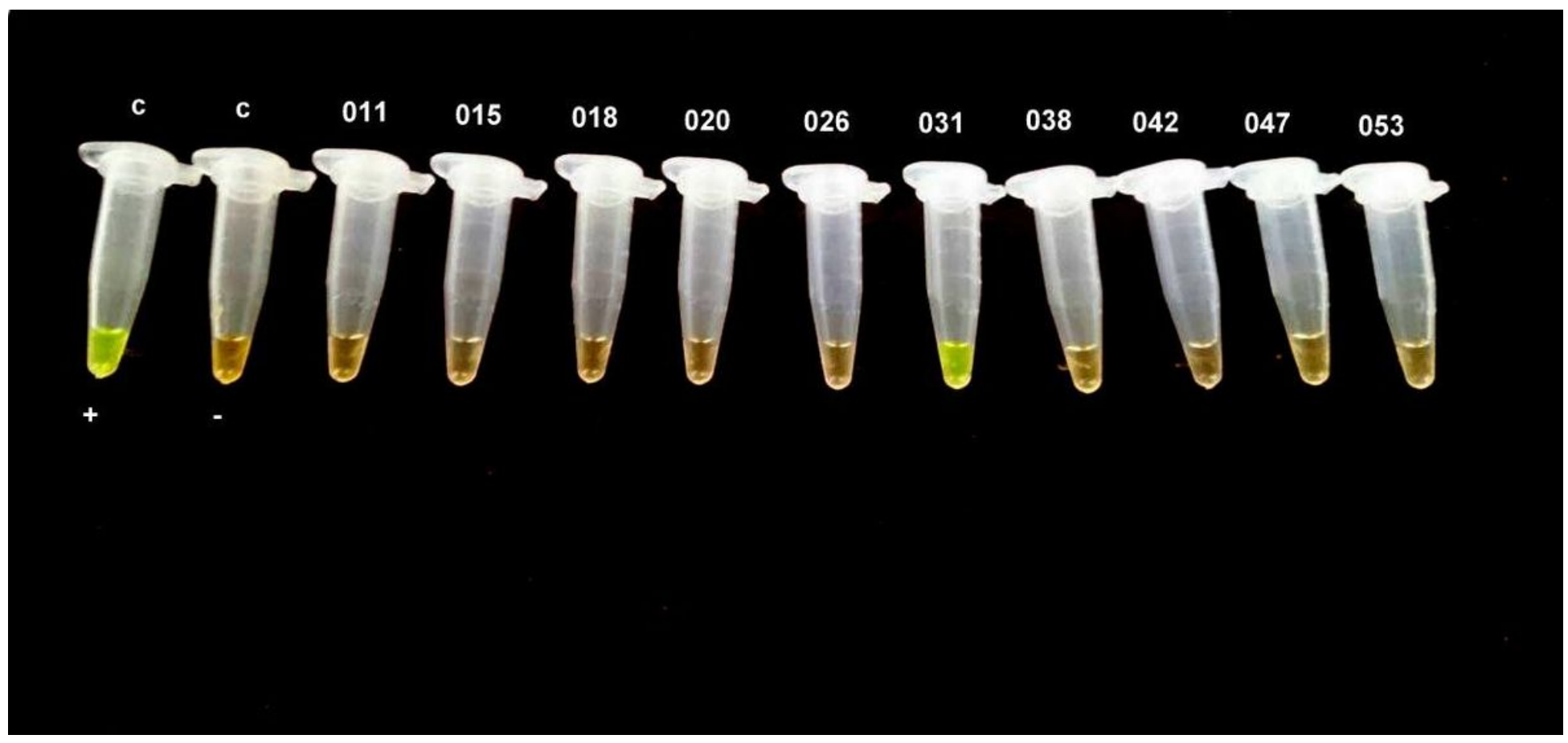

Figure 4

Analysis results of LAMP on stool samples from stray cats using SYBR Green I DNA stain under UV light. C+; Positive control, C-; Negative control, microtube 031; represent the only positive LAMP result for T. cati in the DNA sample from stray cat's feces 


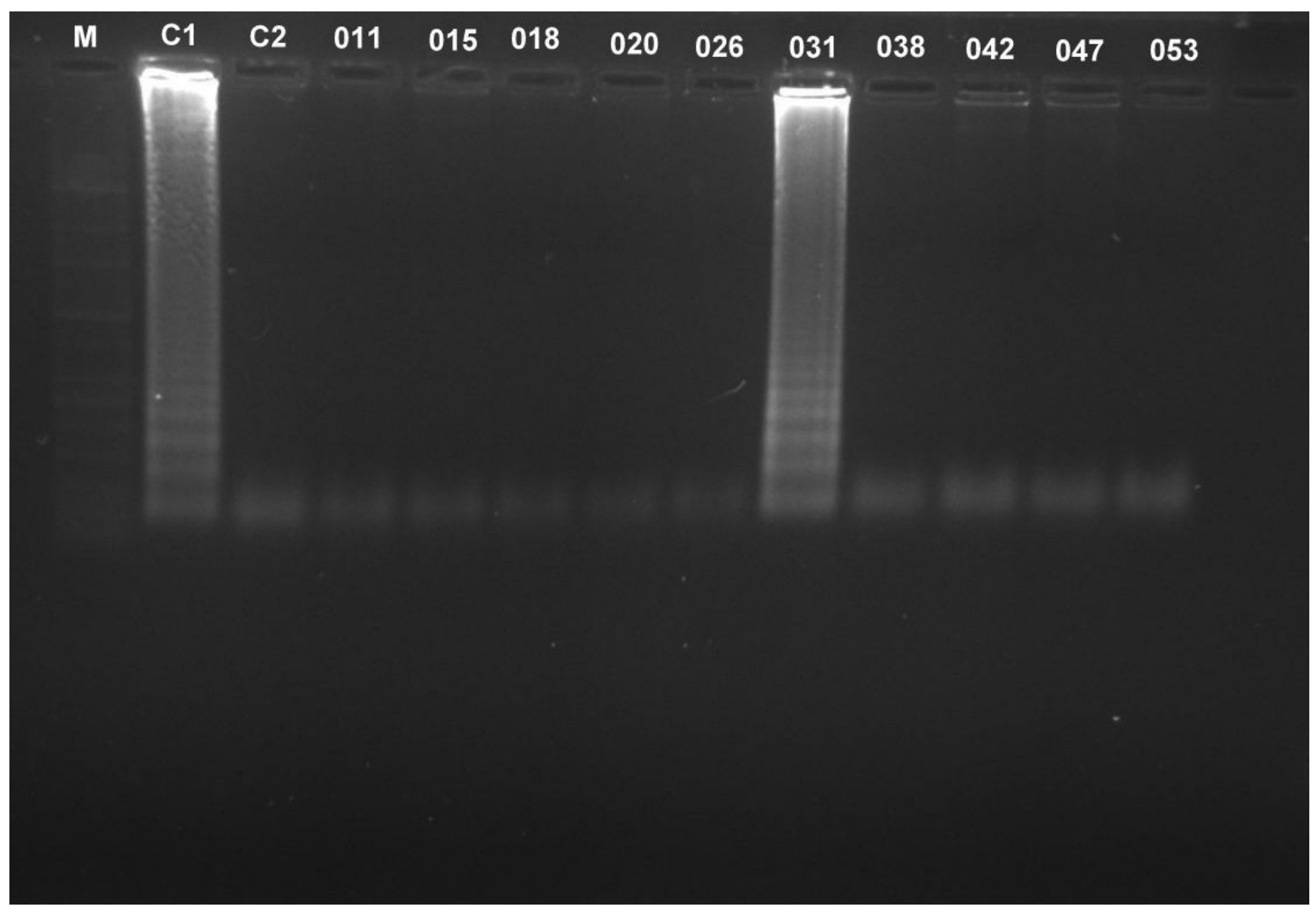

Figure 5

Evaluation of LAMP products by electrophoresis on 1.5\% agarose gel stained with SYBR Safe DNA gel stain. M; 100 bp molecular weight marker, C1; Positive control, C2; Negative control, lane 031; represent the only positive LAMP result for T. cati in the DNA samples from stray cat's feces 